\title{
EDITORIAL
}

\section{Discurso pronunciado por el doctor Fernando Cardona Arango en el homenaje a los expresidentes nacionales, con motivo de los 25 años de la fundación de la Federación Colombiana de Sociedades de Obstetricia y Ginecología.}

En este acto académico que esta noche nos congrega, realmente es oportuno repasar las generaciones mayores y aprender los jóvenes médicos, la evolución histórica de la obstetricia y la ginecología en nuestra muy querida Colombia.

Fue en la década de 1940 cuando por primera vez los médicos obstetras y ginecólogos de las diferentes regiones de nuestro país, sintieron la necesidad de asociarse en sus respectivas ciudades con fines científicos y gremiales, para estudiar problemas que les eran comunes, definir conductas en patologías discutibles referente a los procedimientos más óptimos a elegir, presentar y analizar casos de patologías de una mínima frecuencia, estudiar la eficacia entre nosotros de los nuevos productos terapéuticos y en síntesis, para juntos proceder por el avance científico de la especialidad en Colombia, a semejanza de lo ya establecido en los países australes de Uruguay, Argentina y Chile que ya eran conocidos por algunos de nuestros médicos que a aquellas naciones habían viajado para ampliar sus horizontes académicos.

$Y$ es así como en Bogotá, un distinguido grupo de obstetras orientados por los profesores doctores, José del Carmen Acosta Villaveces; Víctor Domingo Rodríguez Aponte y Ricardo Forero Vélez, además, de los doctores, Hernando Caicedo Díaz, Enrique Hurtado Salazar, Alfredo Pinto Valderrama, Santiago Lleras Codazzi, Ramón Francisco Sánchez, Rafael Ramírez Merchán y Rafael Peralta Cayón entre otros, fundan el 30 de marzo de 1943, una nueva sociedad a la que denominan "Sociedad de Obstetricia de Colombia” y que luego año y medio más tarde se oficializó su constitución en septiembre de 1944.

Por aquella época en la ciudad de Bogotá, a semejanza de lo efectuado por los obstetras, un grupo de ginecólogos crea la "Sociedad Colombiana de Ginecología", encontrándose entre sus fundadores los profesores Arturo Aparicio Jaramillo, Daniel de Brigard Herrera, Hernando Amaya León, Héctor Enrique Bernal y Guillermo López Escobar.

Estas dos sociedades después de un amplio diálogo, resolvieron fusionarse y oficialmente así lo hicieron mediante resolución 253 del 17 de octubre de 1949; esta nueva sociedad se denominó "Sociedad Colombiana de Obstetricia y Ginecología”; su primera Junta Directiva fue integrada por: Rafael Ramírez Merchán, presidente; Jorge Amorocho Carreño, Vice-presidente; Rafael Peralta Cayón, Tesorero; Jaime Correal Maldonado, Secretario general; Guillermo López Escobar, Secretario de actas.

A finales de la década de los 40, se funda en Medellín la Asociación Antioqueña de Obstetricia y Ginecología, por un grupo de profesores que enseñaban estas especialidades en la Universidad de Antioquia, contándose entre ellos, los doctores Nepomuceno Jiménez, Luis E. Abad C., Benicio Gaviria, Jorge Henao Posada y Luis Tirado Vélez, como obstetras; y Pedro Nel Cardona Correa, René Díaz Correa, Gustavo Isaza Mejía y Samuel Isaza Toro, como ginecólogos.

En estos mismos años, en Cartagena de Indias, se funda la Sociedad de Obstetricia y Ginecología de Bolívar, en la Clínica de maternidad Calvo, por los doctores profesores, Teofrasto Tapir; Jorge Milanés Pernes; Napoleón Franco Pareja; Aníbal Perna Mazzeo; Hernando Taylor Henríquez; Jorge A. Calvo y Boris Calvo del Río, entre otros.

En Barranquilla un fenómeno semejante se presenta; se reunen y forman una sociedad científica denominada Sociedad de Obstetricia y Ginecología del Atlántico, entre otros, los doctores: Rafael García Solano, Eloy Barrios Herrera, Fabián Jiménez Arango, Hernando Echeverri Velásquez, Hugo Flórez Moreno y Eduardo Acosta Bendek.

Con la fundación de la Facultad de Medicina, en Santiago de Cali, se constituye la Sociedad Vallecaucana de Obstetricia y Ginecología el 17 de febrero de 1955, por los profesores Jorge Escobar Soto, Jorge Celenilla Mosquera, Alvaro Vallejo, Luis Alfredo González, Libardo Palao, Alonso Trujillo y Oscar Henao, entre otros.

Estas jóvenes sociedades deciden hacer su primera reunión, que tenía un carácter médico quirúrgico, pues se presentaban trabajos científicos y se hacían demostraciones en los quirófanos; se efectuó en Bogotá bajo la dirección del profesor Arturo Aparicio Jaramillo, con la colaboración de los profesores, Pedro Nel Cardona, de Medellín, Jorge Escobar Soto, de Cali; Napoleón Franco Pareja, de Cartagena; Rafael García Solano y Hernando Echeverri Velásquez, de Barranquilla.

A finales de 1955 del 29 de noviembre al 3 de diciembre se efectúa la siguiente reunión de estas sociedades en Santiago de Cali, ya con el nombre de Convención Nacional de Obstetricia y Ginecología, y para el tercer congreso que se efectuó 
en la ciudad de Ibagué en 1957 se habían fundado la Sociedad Caldense de Obstetricia y Ginecología integrada entre otros por los doctores Bernardo Botero Peláez, Oscar Acevedo Ferrer, Jesús Montes Sáenz, Julia Ocampo Avendaño, Mario Calle Mesa y Antonio Giraldo Giraldo. Lógicamente también la Sociedad Tolimense de Obstetricia y Ginecología, integrada entre otros, por los doctores, Jaime Renjifo Pardo; Juan José Arbeláez; Josué Gómez Arbeláez y la doctora Alba Nelly Becerra.

Durante el congreso de Ibagué, por primera vez se plantea la inquietud de denominar a todas estas sociedades como "Sociedad Colombiana de Obstetricia y Ginecología", con el distintivo de la capital del departamento respectivo, como por ejemplo, Sociedad Colombiana de Obstetricia y Ginecología, seccional Cartagena, o Sociedad Colombiana de Obstetricia y Ginecología, seccional Bogotá.

En aquella oportunidad en Ibagué, en 1957, las directivas de Bogotá nos explicó ampliamente como nuestro anhelo no podía volverse realidad, por existir un contrato firmado entre el Instituto Colombiano de los Seguros Sociales de Cundinamarca y los gineco-obstetras de la denominada Sociedad Colombiana de Obstetricia y Ginecología, que tiene su sede en Bogotá.

Y así, lo máximo que se podría lograr era que las nuevas sociedades de provincia figuraran como adherentes de la Sociedad Colombiana de Obstetricia y Ginecología, con sede en la capital de la República.

Todo ésto, con serenidad se oyó, se meditó, deduciendo que en un futuro esta situación era digna de estudio.

Pero, por unos momentos muy breves retrocedamos en el tiempo al mes de marzo de 1949, cuando las directivas de la Sociedad Colombiana consideraron oportuno crear un propio órgano de difusión y para tal fin nombraron al señor Juan $N$. Baquero como administrador de esa nueva publicación periódica que se denominó "Revista Colombiana de Obstetricia y Ginecología"; su primer número se editó en enero de 1950, siendo su primer trabajo científico el del profesor Guillermo López Escobar, con el título de "Algunos aspectos de las cardiopatías y la mecánica circulatoria durante el embarazo". Por gentil invitación de las directivas de Bogotá, las sociedades departamentales que poco a poco se fueron formando, podían publicar, y efectivamente así lo hicieron, sus artículos científicos de la especialidad en esta nueva revista, que así con gran propiedad se podía llamar Revista Colombiana de Obstetricia y Ginecología, no teniendo ello sino una pequeña anormalidad, que consistía que todo el peso de su financiación recaía en la Sociedad Colombiana de Bogotá; las otras sociedades departamentales no contribuían más que con sus aportes científicos y las no muy frecuentes inscripciones voluntarias.

Después del congreso nacional en Ibagué, en 1957, se efectuaron congresos nacionales en Barranquilla, Medellín, Cartagena, y finalmente se programó el próximo congreso en la ciudad de Cúcuta. Previamente en la ciudad fronteriza con Venezuela ya se había constituido la Sociedad Nortesantandereana de Obstetricia y Ginecología, integrada entre otros por los doctores Alberto Duarte Contreras, Humberto Faillace Chiapetta, Eduardo Gómez Franco, Hernando Gómez Sarmiento, Luis Fernando Luzardo, Carlos Fernando Saieh, Ramiro Zúniga Gamboa, Eduardo Pérez Gómez y la doctora Nelly Barbesi Sandoval.

Por estos mismos días, en Bucaramanga se había constituido la Sociedad Santandereana de Obstetricia y Ginecología con los doctores Primitivo Rey Rey, Fabio Durán Velasco, Isaías Arenas Buenahora, Hernán Quijano Mulford, Gonzalo García Gómez y Reynaldo Mora Restrepo, entre otros.

Hasta la fecha, todos estos congresos fueron administrados directamente por la sociedad sede del congreso, pero siempre bajo el patrocinio, supervisión y orientación de las directivas de turno de la Sociedad Colombiana de Obstetricia y Ginecología con sede en Bogotá, que junto con las autoridades locales presidían los congresos.

Estas denominaciones de las sociedades departamentales que francamente no mostraban equidad con la sociedad del departamento de Cundinamarca, creó una cierta inquietud por la desigualdad de su título con respecto al designado por las sociedades de provincia, lo que originó amplios diálogos previos al congreso de Cúcuta, siendo sus principales voceros, los doctores, Fernando del Corral Garcés, por la sociedad Vallecaucana; Eduardo Acosta Bendek, por la sociedad del Atlántico, Alberto Duarte Contreras, por la sociedad Nortesantandereana; Fabio Durán Velasco, por la sociedad Santanderana; Antonio Soto Yances, por la sociedad de Bolívar; y Fernando Cardona Arango, por la sociedad Antioqueña; todos ellos tuvieron amplios y cordiales coloquios con las directivas de la Sociedad Colombiana de Bogotá cuyos voceros fueron los doctores Roberto Vergara Támara, su presidente y Ricardo Rueda González, su secretario.

Para esta época existían las sociedades de Montería y Popayán. En Montería la Sociedad de Obstetricia y Ginecología de Córdoba la constituían entre otros, los doctores, Ricardo Bechara Zainun, José María Cabrales, Eduardo Baquero Betín, Carlos Flores Gómez y Eliécer Arrieta Arteaga, Julio César Zapateiro, Amaury García Burgos y la doctora Inés Botero de Bejarano. 
En Popayán se había fundado la Sociedad Caucana de Obstetricia y Ginecología, entre otros, por los doctores Julio César Payán, Luis Félix Muñoz, Iván Jaramillo Arias, Iván Enrique Fernández, José Vicente Erazo Domínguez, Héctor Suárez Cleves y la doctora Renate Susmann Lewin.

En Sincelejo en el año de 1967 se fundó la Sociedad de Obstetricia y Ginecología de Sucre entre otros por los doctores, Francisco Vergara Villareal, Fredy Jaraba Romero, Hilario Daza Padilla, Humberto Blanco Wilches y Julio García Marichal.

Durante la segunda jornada del congreso, en la noche del 4 de diciembre, en los amplios salones del Club del Comercio de la ciudad de Cúcuta, se reunieron todas las juntas directivas de las sociedades existentes en el país y después de una reunión amable, científica, sincera y plena de una gran discreción, que duró más de tres horas, se llegó a la conclusión por unanimidad, que era necesario y beneficioso para la especialidad el efectuar oficialmente al día siguiente a las 12 del día, sobre las ruinas del templo de la Villa del Rosario, la fundación de la Federación Colombiana de Sociedades de Obstetricia y Ginecología, también denominada FECOLSOG y así en forma muy solemne ese 5 de diciembre de 1967 se llevó a cabo la fundación tan anhelada por más de dos lustros.

Esta primera Junta Directiva para el período 1967-1969 quedó integrada por: Presidente, Roberto Vergara Támara, de Bogotá; Vice-presidente, Fernando Cardona Arango, de Medellín; Secretario, Ricardo Rueda González, de Bogotá; Fabio Durán Velasco, de Bucaramanga; Vocal, Alberto Duarte Contreras, de Cúcuta; Vocal, Eduardo Acosta Bendek, de Barranquilla; Vocal, Fernando del Corral Garcés, de Cali.

Con la constitución de FECOLSOG se lograron importantes objetivos estudiados ya previamente por sus fundadores, como lo es el de la igualdad dentro de la Federación - con los mismos derechos y deberes- de todos sus miembros, ya fuesen ellos de la capital de la república o de la más alejada de las provincias; se logró contar con un número considerable de afiliados, lo que produjo una imagen fuerte y vigorosa de Colombia ante la "Federación Latinoamericana de Sociedades de Obstetricia y Ginecología" denominada FLASOG y cuyos diplomas que así lo acreditan les fue entregado personalmente a todos los miembros por el señor Presidente de FECOLSOG en el período 77-79, cuando visitó a cada una de las sociedades.

En el campo mundial los miembros de FECOLSOG, por resolución estatutaria, entraron a formar parte de la Federación Internacional de Obstetricia y Ginecología o también denominada FIGO cuyo diploma de su respectiva credencial fue entregado también en 1979 por el señor Presidente al regreso del congreso de Tokio; estos diplomas llevan la firma del señor Presidente de la FIGO en ese período, el profesor Roberto Caldeyro-Barcia para mayor orgullo nuestro.

Otro de los logros obtenidos con la fundación de FECOLSOG fue la adquisición de un órgano propio de difusión científica, y al efecto se establecieron conversaciones con las directivas de la Sociedad Colombiana y en esta forma se logró amistosamente que la mitad de la revista pasara a ser propiedad de la Federación, la que de inmediato se encargó de la mitad de su financiación y por estatutos hizo efectivo que todos sus miembros fuesen obligatoriamente suscriptores de la Revista Colombiana de Obstetricia y Ginecología y nombró un co-director el cual es renovado periódicamente. En la actualidad sus directores son por la Sociedad Colombiana el doctor Gilberto Martínez Morales y por FECOLSOG el doctor William Onatra Herrera.

Con positivo orgullo podemos resaltar que en la actualidad es la publicación científica médica más antigua del país, que no ha presentado ni un solo período de interrupción, en sus 42 años de existencia y que sus directivas todas han sido de una laboriosidad ejemplar, como lo son sus actuales dirigentes; pero no podemos continuar en este tema, sin mencionar algunos de sus exdirectores, como son los doctores Francisco Pardo Vargas, José Gabriel Acuña Díaz, Enrique Archila Aguilera, Jesús Alberto Gómez Palacino, Miguel Alvaro Fernández Bastidas, Fernando Sánchez Torres, Hernando Amaya León, Germán Uriza Gutiérrez y el ya mencionado anteriormente, Guillermo López Escobar.

Creemos con sobrada razón que son ciertas y actuales las palabras del Presidente de la Federación cuando se celebró el congreso en Bogotá en 1979 al referirse a la revista y decir que ella era la joya más valiosa que poseía FECOLSOG.

Durante estos 25 años, FECOLSOG ha trabajado intensamente en el campo nacional, promocionando los congresos nacionales, como el efectuado en Pereira por la Sociedad Pereirana, con el doctor Ariel Orrego Gómez como presidente y con la colaboración de los doctores Fabio Sanz Hurtado, Fernando Ramírez Alvarez, Abel Villegas Botero, Rafael Orrego Herrera y la doctora Amanda Vélez. FECOLSOG ha proporcionado ayuda económica para iniciar labores a la Junta Directiva de la ciudad sede del próximo congreso, orientando a los comités científicos, en la elección de los seminarios y velando por los tiempos suficientes para los temas libres que tan importantes son, pues son sus autores los investigadores y los profesores del mañana.

También ha prestado una valiosa ayuda en la programación y elaboración de cursillos regionales y ha logrado fundar nuevas sociedades departamentales como la Sociedad Nariñense y la Sociedad del Cesar y ha revitalizado sociedades que prácticamente se habían extinguido, como la Sociedad del Quindío, la del Magdalena y la Huilense. 
En el período presidencial del doctor Oscar Acevedo Ferrer, se efectuó una asamblea extraordinaria, se reformaron los estatutos y se aprobaron los nuevos reglamentos en lo referente a la metodología de los congresos. En dicha asamblea que se celebró en el Hotel Intercontinental en Medellín, se creó un nuevo cargo en la Junta Directiva y fue el del Fiscal, siendo el doctor Hernán Restrepo Ramírez, de Cali, nombrado inicialmente para este cargo.

En la presidencia del doctor Antonio Soto Yances, se hizo el primer trabajo científico remunerado, con el Ministerio de Salud, que lo efectuó el doctor Francisco Pardo Vargas y col. presentado en el Congreso de Bucaramanga. Posteriormente, en la presidencia del doctor Roberto Jaramillo Uricoechea, se efectuó el segundo trabajo remunerado en el Ministerio de Salud, en el tópico de la historia perinatal. Estos trabajos lograron que FECOLSOG no dependiera para su subsistencia sólo de los ingresos de las inscripciones de los congresos y que pudiera así dar una amplia colaboración a la financiación de la Revista. En este campo el último aporte de consideración lo obtuvo el señor presidente Francisco Pardo Vargas, con el secretario de la Federación, doctor Germán Uriza Gutiérrez, y son dos trabajos de investigación remunerados patrocinados con capital extranjero que fueron presentados en el Congreso Latinoamericano de la FLASOG en Montevideo, estos trabajos con investigaciones multicéntricas de los cinco países han sido los únicos efectuados en los grupos Latinoamericanos hasta el presente.

FECOLSOG ha tenido un papel preponderante en su relación con la FLASOG, al crear bajo la presidencia del doctor Roberto Jaramillo Uricoechea, el denominado "Grupo Bolivariano" compuesto por los países de Bolivia, Perú, Ecuador, Venezuela y Colombia, que tuvo su primera reunión en La Paz y al organizar posteriormente en Bogotá, bajo la presidencia del doctor Francisco Pardo Vargas, la segunda reunión del grupo bolivariano con tan brillante éxito en su trabajo de conjunto sobre el tema del embarazo en la mujer adolescente, que por unanimidad de los delegados pasó a liderar FECOLSOG el grupo bolivariano de la FLASOG y se creó la sede permanente de este grupo en la tradicional Santafé de Bogotá.

La FLASOG ha tenido dos nombramientos que son altamente honoríficos para FECOLSOG. El uno, haber tenido durante varios años como secretario general de la Federación Latinoamericana al profesor Fernando Sánchez Torres y el otro el haber nominado como parte esencial del comité científico del próximo congreso de celebrarse en Panamá, al profesor Francisco Pardo Vargas. FECOLSOG se siente muy orgullosa de estos dos nombramientos.

En el campo mundial en relación con la FIGO, poca ha sido la influencia que ha desarrollado FECOLSOG en sus actividades científicas que durante años se han limitado a la participación en los congresos mundiales en los temas libres; no obstante, merece destacarse una de las cuatro conferencias magistrales del congreso de San Francisco que fue valientemente expuesta en español en forma brillante por el expresidente, profesor Edgar Cobo Cobo, que dicho sea de paso domina perfectamente el inglés; también en este congreso presentó Colombia por primera vez un seminario correlacionado sobre Patología Cervical Maligna en el Embarazo, y el trabajo fue elaborado por los expresidentes, Fernando Cardona Arango y Antonio Soto Yances, en asocio con el actual presidente, Jaime Barrios Amaya; cabe mencionar que el doctor Edgar Cobo Cobo, formó parte del comité científico durante este período de la FIGO, en que se efectuó el congreso de San Francisco.

La Federación ha contado desde su fundación con seis presidentes de Santafé de Bogotá, que son los profesores doctores, Roberto Vergara Támara; Jesús Alberto Gómez Palacino; Hernando Navas Angel; Eduardo Cáceres Alvarez; Roberto Jaramillo Uricoechea y Francisco Pardo Vargas; con dos presidentes de Cartagena, los profesores doctores; Antonio Soto Yances y Jaime Barrios Amaya su actual presidente; con uno de Barranquilla, el profesor doctor Eduardo Acosta Bendek; con uno de Manizales, el profesor doctor Oscar Acevedo Ferrer; con uno de Santiago de Cali, el profesor doctor Edgar Cobo Cobo; y con uno de la ciudad de Medellín, el profesor doctor Fernando Cardona Arango.

Finalmente, si analizamos el presente de FECOLSOG, vemos que tiene en la actualidad un aceptable estado financiero que permite cumplir con los compromisos de la revista, con las cuotas de afiliación a la FIGO y a la FLASOG, con los aportes iniciales a los congresos próximos y con los gastos de secretaría, los costos de las reuniones de la Junta Directiva y los costos a la asamblea general de delegados.

Vemos que en el campo científico continúan los trabajos de investigación remunerada, se adelantan jornadas científicas tan brillantes como las que estamos viviendo en estos días y se orientan, preparan y coordinan las labores del próximo congreso nacional; vemos que en la actualidad se ha programado en forma casi vertiginosa en el campo laboral, que se habían manejado en forma muy discreta, casi mínima, pero que con la problemática de la medicina prepagada y con los nuevos horizontes de la responsabilidad civil de los procedimientos médico-quirúrgicos, la federación ha tomado un puesto de avanzada en los estudios pertinentes para buscar y obtener protección justa y responsable de todos los miembros de FECOLSOG, como muy claro se encuentra en los estatutos en el capítulo 2o., ordinales $M$ y $N$.

Presenta en la actualidad la Federación un pequeño problema con la denominada Sociedad Colombiana de Obstetricia y Ginecología, que en el fondo no es más que un problema de semántica, pues esta sociedad que fue la primera que se fundó en el país, no debería en el sentido estricto actual denominarse Colombiana ya que por estatutos que esta misma 
sociedad aprobó no puede existir más de una sociedad por departamento, intendencia o comisaría del país (artículo 8 del capítulo 3 de los estatutos de la Federación) y estamos íntimamente convencidos de que la forma correcta sería su denominación, Sociedad de Cundinamarca o Sociedad de Santafé de Bogotá como Distrito Especial, pues en forma geográfica su área de acción queda circunscrita a los límites departamentales de Cundinamarca.

En la evolución histórica de las sociedades nacionales, este fenómeno que hemos denominado en este caso de semántica que los gineco-obstetras vivimos, espero que por muy poco tiempo, también se presentó en igual forma en la especialidad de pediatría en donde fue discutido en una asamblea general en forma un poco exaltada pero en donde finalmente, el análisis frío de los argumentos expuestos y una buena dosis de gran cordialidad y con un alto espíritu de unión, la sociedad de pediatría de la capital de la república cambió su nombre de Colombiana por el de Santafé de Bogotá; estoy seguro que estos 25 años que celebramos con orgullo y alegría servirá como aliciente para que las directivas y todos sus miembros en Santafé de Bogotá, siguiendo el noble ejemplo de la sociedad de pediatría, reflexionen sobre este tópico y prontamente procedan al cambio de su denominación, como todo el país lo espera.

Hemos tratado de efectuar en una forma francamente sucinta la historia de la Obstetricia y Ginecología en Colombia en los últimos 50 años y como en un momento dado se fundó la Federación Colombiana de Sociedades de Obstetricia y Ginecología, FECOLSOG.

Nos hemos referido en más de una ocasión a varios maestros que sin duda alguna fueron los pilares fundamentales de la moderna obstetricia y ginecología, pero ellos ya se han ido para siempre y no nos queda más que el recuerdo de los que fueron nuestros profesores; nos alienta sin embargo el que sus enseñanzas continúan presentes a través de nosotros sus discípulos y que por medio de la Federación les transmitimos a las nuevas generaciones lo que de ellos aprendimos, junto con nuestros nuevos conocimientos y experiencias.

Estas jóvenes generaciones en un tiempo más o menos corto tomarán nuestros puestos de dirigentes de la gineco-obstetricia colombiana y serán los que en cinco lustros celebrarán los 50 años de FECOLSOG y confiamos que por nuestras obras nos recordarán.

Honor y gloria a todos esos científicos estudiosos y consagrados maestros pioneros que nos han precedido. Honor y gloria a todos los expresidentes de FECOLSOG que con su entrega sin limitaciones y su acertada dirección tienen a la Federación en tan alto lugar en el ámbito nacional e internacional. Y honor y gloria a todos los miembros actuales de FECOLSOG que con su presencia física y su apoyo científico hacen que todos nosotros con una gran fe y un gran optimismo nos preparemos a saludar el futuro.

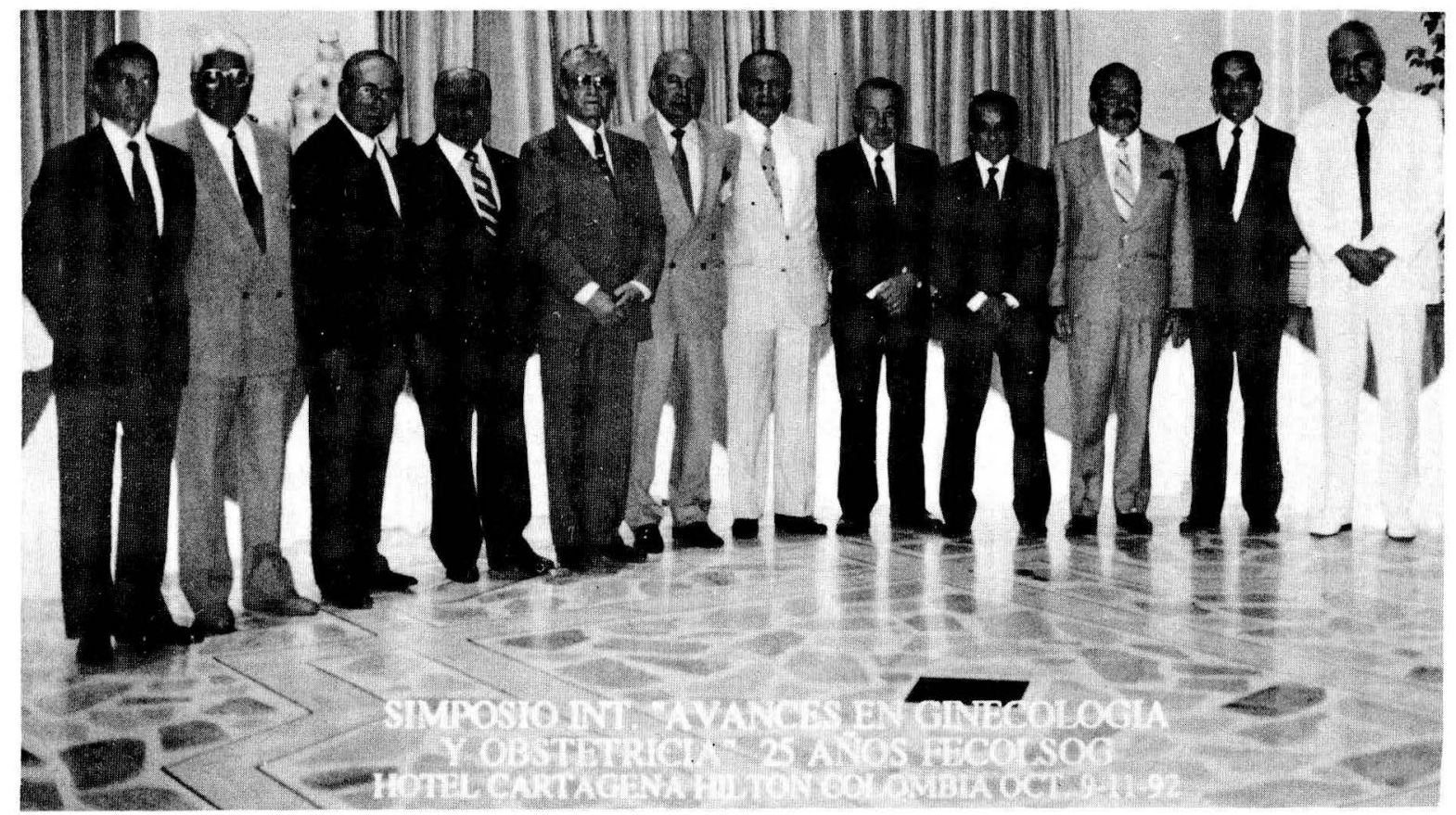

Francisco Pardo Vargas, Roberto Jaramillo Uricoechea, Fernando Cardona Arango, Eduardo Cáceres Alvarez, Oscar Acevedo Ferrer, Roberto Vergara Támara, Eduardo Acosta Bendek, Hernando Navas Angel, Jesús A. Gómez Palacino. Edgard Cobo Cobo, Antonio Soto Yances y Jaime Barrios Amaya. 


\title{
fundación de la Federación Colombiana de Sociedades \\ de Obstetricia y Ginecología.
}

\section{Discurso pronunciado por el Dr. Jaime A. Barrios Amaya, presidente de Fecolsog, con motivo del simposio "Avances en Ginecología y Obstetricia” 25 Años Fecolsog.}

\begin{abstract}
Nos hemos reunido con motivo de la celebración de los 25 años de fundada la Federación Colombiana de Sociedades de Obstetricia y Ginecología, que logró aglutinar a las diferentes Sociedades de los departamentos colombianos en 1967, en la bella ciudad de Cúcuta.

Contamos con la presencia de destacados profesionales del país y del exterior, quienes amablemente respondieron a nuestra invitación y serán los ponentes de un variado y actualizado temario de mucho interés para médicos generales, estudiantes, enfermeras y especialistas.
\end{abstract}

Los médicos, y especialmente los ginecólogos, tenemos un compromiso con el bienestar de nuestra comunidad, de nuestro país, de toda América y del mundo entero. El Todopoderoso nos ha dado la oportunidad de servir y ayudar al ser más preciado sobre la tierra, como lo es la MUJER.

Las instituciones de salud y nuestras acciones deben estar encaminadas a proteger a las mujeres desde la vida fetal hasta la vejez. Llevar a cabo programas de prevención de enfermedades infecto contagiosas, virales y de transmisión sexual. Hacer campañas de vacunación y de prevención del cáncer genital y mamario. Continuar nuestras labores en Planificación Familiar. No podemos aceptar que, porque nuestro país tiene un bajo índice de crecimiento demográfico, se disminuyan las campañas de planificación familiar y Salud Reproductiva. Las estadísticas son incompletas y viciadas a este respecto: porque mientras una familia de clase alta o media alta sólo desea tener dos hijos, la gran población de clase baja, por ignorancia e incomunicación, tiene muchos hijos viviendo en hacinamiento y promiscuidad. Nuestra obligación es desarrollar campañas educadoras en los cinturones de miseria de las grandes ciudades y en los pequeños municipios y corregimientos donde la pobreza es mayor.

Debemos preservar y mantener la salud de la madre para evitar las altas tasas de mortalidad materna que aún se registran en los países más deprimidos de Latinoamérica. Propiciar la llegada al mundo de un recién nacido sano para que su desarrollo y crecimiento sean normales. Guiar a las adolescentes en su despertar sexual para evitar embarazos no deseados, y vernos frente a la grave situación de adolescentes pobres, desposeídas y embarazadas.

La salud de la mujer es nuestra gran meta y así como luchamos porque una familia crezca en función de sus recursos económicos y culturales, también debemos poner al servicio de las parejas infértiles todos nuestros conocimientos y tecnología moderna para verlas sonreír y gozar ante la llegada de un hijo que creían imposible de gestar.

Debemos continuar nuestra acción, al conservar la salud de la mujer climatérica y post menopáusica. Los adelantos farmacológicos están dando la gran oportunidad a las mujeres de una vejez feliz y sana, porque desde los 50 años de edad, $o$ antes, están recibiendo el tratamiento adecuado, la dieta propicia, y las recomendaciones de ejercicios físicos y contacto sano con el medio ambiente. Si la expectativa de vida de las mujeres está aumentando en toda Latinoamérica, ésta mayor oportunidad de vivir debe ser sin "sofocos" ni osteoporosis.

Queremos destacar la presencia de los 11 expresidentes de FECOLSOG, quienes han sido los forjadores de esta sólida institución científica, que ha venido durante 25 años haciendo patria a través de congresos, seminarios, coloquios y reuniones, para beneficio del país.

Agradecemos sinceramente la respuesta de ustedes a este Simposio de Avances en Ginecología y Obstetricia y en especial a las delegaciones de Panamá y Costa Rica.

Doy gracias a Dios por estar aquí, unos aprendiendo y otros enseñando, bajo la guía científica del Maestro de Maestros, Profesor Roberto Caldeyro- Barcia, y del Profesor Alfredo García, en esta bella ciudad de Cartagena, al suave rumor de las palmeras y las azules olas marinas, y como dijera mi vado de cabecera, el Tuerto López: Cartagena, Arcadia del Caribe... plena de rancio desaliño, bien puedes inspirar ese cariño, que uno les tiene a sus zapatos viejos".

Gracias.

Cartagena, 9 de octubre de 1992. 